\title{
Ongoing primary care intervention increased remission and emotional and physical role functioning in major depression
}

\author{
Rost K, Nutting P, Smith JL, et al. Managing depression as a chronic disease: a randomised trial of ongoing treatment in \\ primary care. BMJ 2002;325:934-7.
}

\section{QUESTION: In patients with major depression, is ongoing intervention (augmented care management) more effective than usual care for increasing remission and emotional and physical role functioning?}

Sources of funding: National Institute of Mental Health and the John D and Catherine TMacArthur Foundation.

For correspondence: Dr K Rost, Department of Family Medicine, University of Colorado Health Sciences Center at Fitzsimons, Aurora, CO, USA.

Kathryn.Rost@

UCHSC.edu

\section{Design}

Cluster randomised (allocation concealed), blinded (data collectors), controlled trial with 24 months of follow up.

\section{Setting}

12 primary care practices (8 in metropolitan and 4 in non-metropolitan areas) in the US.

\section{Patients}

211 patients (mean age $43 \mathrm{y}, 85 \%$ women) with major depression (defined as reporting $\geq 5$ of 9 criteria for

Enhanced v usual care for major depression at 24 month*

\begin{tabular}{lllll}
\hline Outcomes & Enhanced care & Usual care & RBI (95\% Cl) & NNT (Cl) \\
$\begin{array}{l}\text { Patients in } \\
\text { remission }\end{array}$ & $74 \%$ & $41 \%$ & $82 \%(41$ to 140$)$ & 4 (3 to 5) \\
$\begin{array}{l}\text { Emotional role } \\
\text { functioning (mean } \\
\text { MCES-D points) }\end{array}$ & 73 & 49 & 24 (11 to 38) \\
\hline $\begin{array}{l}\text { Physical role } \\
\text { functioning (mean } \\
\text { MCES-D points) }\end{array}$ & 61 & 44 & 17 (6 to 28) \\
\hline
\end{tabular}

*MCES-D = modified Center for Epidemiological Studies-depression. Abbreviations defined in glossary; RBI, NNT, and $\mathrm{Cl}$ calculated from data in article. Data available from all 4 time points were used in intention to treat analy-

\section{COMMENTARY}

Depression continues to be a primary public health concern and drain on limited healthcare resources despite the availability of highly effective means of treatment. Factors influencing the continuing prevalence of depression may, in part, be because of the chronic and recurrent nature of this illness. The chronicity and recurrence of depression are receiving increased recognition and attention by clinicians and researchers alike because these challenging features offset the initial gains from treatment.

The trial by Rost $e t$ al is important because it tests interventions that target the period of time after the initiation of treatment for depression. With a minimal amount of adjunct education, nurses working in healthcare clinics and offices offer enhanced follow up care for patients who have just begun treatment for depression. These interventions would encourage and empower patients to be active in their recovery process using the specially trained nurse care manager as a source of support and knowledge. This study shows that enhanced care substantially improved depression treatment effectiveness by increasing patient remissions and physical and emotional well being.

Although the number of patients in the treatment and control groups was relatively small for a quantitative study, the intention to treat analysis used all the data collected at various time points, which adds strength to the study. The significant difference in outcomes suggests that enhanced follow up care by nurses is a valid and feasible means of sustaining clinical improvements in the longer term for patients at risk of recurrence for depression. Nurses encourage, empower, support, and educate in all practice settings, which makes this intervention a natural and effective extension of the nursing process.

Deborah Kindy, RN, PhD

Associate Professor

Department of Nursing, Sonoma State University Rohnert Park, California, USA major depression listed in the Diagnostic and Statistical Manual of Mental Disorders, 3rd edition revised) who where identified after screening during routine visits to primary care practices. Exclusion criteria included bereavement, mania, alcohol dependence, pregnancy or postpartum period, or life threatening physical illness. Patients who did not intend to use the clinic as their usual source of care during the year after the index visit were also excluded. Follow up was 90\%, 82\%, 73\%, and $67 \%$ at $6,12,18$, and 24 months, respectively.

\section{Intervention}

The 12 primary care practices were matched into 6 blocks by pairing practices with similar proportions of patients with depression who were receiving care in accordance with guidelines. Subsequently, 6 primary care practices each (block by block) were allocated to enhanced care $(n=115)$ or usual care $(n=96)$. Participating physicians, nurses, and support staff in the enhanced care group received training designed to encourage patients to select and maintain ongoing treatment based on the Agency for Health Care Policy and Research guidelines. The initial intervention (baseline to $6 \mathrm{mo}$ ) was aimed at increasing the number of patients who received pharmacotherapy or psychotherapy, and continuing intervention (7-24 mo) was aimed at sustaining or increasing improvement. In the usual care group, patients received no regular contacts from nurse care managers throughout the study.

\section{Main outcome measures}

Patient reports of remission (modified Center for Epidemiological Studies-depression scale equivalent to a score $<16$ ) and functioning (using two 100 point subscales of Short Form-36 that examine perceived limitations in usual daily activities in the previous month because of physical and emotional problems, with higher scores indicating improved outcomes).

\section{Main results}

Analysis was by intention to treat. The enhanced care group had greater increases from baseline in rate of remission and emotional and physical role functioning than the usual care group throughout follow up ( $p$ values $<0.05$ ) (table).

\section{Conclusion}

In patients with a recent diagnosis of major depression, ongoing intervention was more effective than usual care for improving symptom remission and emotional and physical role functioning. 